\title{
Atoms Through the Looking Glass - A Relativistic Challenge
}

\author{
Ann-Marie Mårtensson-Pendrill
}

\begin{abstract}
:
The search for weak-interaction induced atomic parity non-conservation, initiated in the 70 s, challenges both theory and experiment. Since the weak interaction is very short-range, the atomic effects increase rapidly with nuclear charge, as $Z^{3}$. The focus has thus been on heavy atoms, where relativistic effects are essential, and nuclear size must be taken into account. The generalization of atomic many-body methods to relativistic systems involved both computational and formal difficulties, incorporating methods developed in quantum electrodynamics. Twenty years ago, the ability was emerging to treat atomic pair correlation in a relativistic framework. The application to many-electron systems opened for comparison with many atomic properties, such as isotope shifts, hyperfine structure and hyperfine anomalies, which reflect nuclear properties. In addition, the search for simultaneous violation of both parity and time reversal symmetry involves different types of effects, including nuclear "Schiff moments". Comparison between computed and experimental results for highly charged hydrogen-like systems provides a test of the theoretical and numerical treatment of the electron-nucleus interaction and of the description of nuclear distributions.
\end{abstract}

Key words: Bohr-Weisskopf effect, nuclear distribution, magnetization, hyperfine anomaly, isotope, parity, edm, relativistic pair correlation.

\section{Introduction}

The 1956-1957 Parity Revolution [1, 2] shook physicists' belief in symmetries, so strongly taken for granted that mirror symmetry had hardly been tested [3]. The fifty year anniversary of the Parity Revolution coincides with the EBIT twenty year celebration. This paper presents a broad view of the development of atomic theory over two decades, in connection with the quest for atomic parity non-conservation (PNC), which challenged the development of methods to treat pair correlation for relativistic systems. The road to calculations of PNC effects also passes calculations of other properties involving the atomic nucleus, such as hyperfine structure, isotope shift and hyperfine anomalies.

The combination of atomic theory with experimental results can give information about nuclear distributions, which may be relevant for the study of atomic parity non- conservation, with and without accompanying violation of time reversal symmetry. An EBIT-related example is the calculation of the Bohr Weisskopf effect in $\mathrm{Tl}$ in connection with measurements by Peter Beiersdorfer and colleagues [4].

Ann-Marie Mårtensson-Pendrill. Department of Physics, Göteborg University

SE-412 96 Göteborg, Sweden e-mail: Ann-Marie.Pendrill@ physics.gu.se 


\subsection{Asymmetries in Nature}

In our everyday life, there are many examples of differences between left and right, not only in human constructions. If you find a seashell at the beach the probability that it is right-handed is overwhelming, even on the southern hemisphere. Honeysuckles are lefthanded. Nearby right-handed examples are found in the Berkeley campus eucaluptus trees.

The asymmetries occur also on a smaller scale. The double helix of the DNA, e.g., is always righthanded. Early investigations of the nature of asymmetric molecules were performed by Pasteur [5]. He found that a solution of sugar was optically active, in contrast to substances he could synthesize in the laboratory. Using tweezers under microscope, he then sorted crystals of tartaric acid, and dissolved the two types separately - and found that one of the solutions gave an optical rotation to the right, whereas the other solution gave an equal, but opposite, rotation. Pasteur was fascinated by the asymmetry, and thought that it might include the key to understanding life. In an effort to create an unbalanced mixture of right- and lefthanded molecules, he tried to apply a strong magnetic field; we all remember the right-hand rules for electromagnetism. Still, Pasteur was unsuccessful. Electromagnetism is symmetric under mirror inversions. Every physical effect requires two applications of a right-hand rule. Thus, replacing the right-hand rule by the left-hand rule of the mirror does not change the physical outcome of electromagnetic interactions.

\subsection{Mirrors, Inversions and Rotations}

A left-handed spiral becomes right-handed on the other side of a mirror, but no amount of rotation can change its handedness. A 180-degree rotation is equivalent to an inversion of two coordinate axes. A second inversion would return the original handedness. A Parity inversion changes all three coordinate axes, and is thus equivalent to a mirror inversion followed by a 180 degree rotation.

Until 50 years ago most physicists took for granted that the fundamental interactions were mirror symmetric, and thus unchanged by a parity inversion. When Lee and Yang proposed that parity might be violated in beta-decay [1], Richard Feynman bet Norman Ramsey 50 dollars to 1 that physics in the mirror world would be unchanged. He lost. The now classical experiment by Wu et al showed that the beta decay of ${ }^{60}$ Co violated parity [2]. Ramsey resumed the search, initiated already in 1950 [3], for a neutron electric dipole moment, which would violate parity. This seems to have been the first questioning of the parity conservation.

\subsection{Atomic Parity Non-Conservation}

Weak interactions become relevant for atomic physics through the neutral current component. The neutral vector boson, $\mathrm{Z}$, was discovered in CERN in 1983, at about the same time as the charged boson W. The vector bosons mediate the weak interaction, and are exchanged as virtual particles. The weak interaction is very short range since the mediating bosons are heavy (the mass of the $\mathrm{Z}$ boson is comparable to a $\mathrm{Sr}$ atom) and cannot go far before annihilation. It influences the electron-nucleon interaction, giving it a parity non-conserving part, which leads to a weak chiral component in the electron wavefunction. This makes atoms optically active. The nuclear spin-independent part of the resulting parity non-conserving interaction can be written as:

$h^{P N C}=\frac{G_{W} Q_{W}}{2 \sqrt{2}} \rho(r) \gamma_{5}$.

Here, $G_{W}$ is the weak interaction constant and $Q_{W} \approx-N+Z\left(1-4 \sin ^{2} \theta_{W}\right)$ is the "weak nuclear charge", which depends on the strength of the weak interaction parameters. The short-range character of the interaction is indicated by $\rho(r)$, which is a normalized nuclear distribution, essentially following the neutron distribution. The parity admixture is caused by the operator $\gamma_{5}$, which interchanges the upper and lower component of a relativistic electron wavefunction. 
For a long time, it was believed that the effect of the weak interaction was too small to be observed. The quest for atomic parity non-conservation was initiated by Bouchiat and Bouchiat [6] who pointed that the effect is strongly enhanced for heavy atoms, displaying a $Z^{3}$ behaviour. By using semiempirical methods they could demonstrate that it might be observable for heavy atoms. They suggested a few candidate atoms, such as $\mathrm{Cs}, \mathrm{Tl}, \mathrm{Pb}$ and $\mathrm{Bi}$. During the years to come, experiments were undertaken at a number of laboratories, including Paris, Oxford, Seattle, Novosibirsk, Berkeley and Boulder. After initial years of confusing and contradicting results, the effect is now well established. The most precise results have been obtained for $\mathrm{Cs}(\mathrm{Z}=55)$, where the experimental accuracy is at the $0.4 \%$ level $[7,8]$. These results challenge theoretical calculations [9, 10]. At this level of accuracy, a careful treatment of the Breit interaction [11,12] and QED effects [13] is necessary for agreement between theoretical and experimental data $[11,12]$.

As a method to circumvent the need for accurate atomic calculations, it has been suggested [14] that measurements of parity non-conservation be performed over chains of isotopes, under the assumption that the atomic factor would be the same for all isotopes. The dependence on the weak charge could then be factored out. A complication, which limits the attainable accuracy, as pointed out by Fortson $e t$ al $[15,16]$, is that the neutron distribution is, in general, not known, in contrast to proton distributions which have long been studied, e.g. through isotope shifts in atomic transitions. James and Sandars [17] performed a parametric analysis of the sensitivity, both to changes in the charge radii and to differences between neutron and proton radii.

The weak interaction between nucleons may also give rise to a parity non-conserving nuclear anapole moment $[18,19]$. This combines with the spin-dependent part of the electron-nucleon parity nonconserving interaction. This results in a hyperfine dependence which has been measured for Cs [20], and experiments are underway for other systems, including Fr [21].

\subsection{Electric Dipole Moments and Time Reversal Violation}

"The argument against electric dipoles raises directly from the question of parity. A nucleus with an electric dipole moment would show an asymmetry between left- and right-handed coordinate systems. In one system the dipole moment would be parallel to the angular momentum and in the other antiparallel.", Ramsey and Purcell write in a 1950 paper [3] and continue by noting that "the question of a possible existence of an electric dipole moment of a nucleus or of an elementary particle [...] becomes a purely experimental matter".

Although the existence of parity violating interactions is well established, the experimental search for a possible neutron electric dipole moment (EDM) continues to provide only more and more stringent upper limits. An EDM violates not only parity, but also symmetry under time reversal [22], (most easily visualized by reversing all motions). CP violation, which implies $\mathrm{T}$ violation if CPT is conserved, was discovered in the decay of neutral K mesons in 1964 [23]. The ever-falling limits of the EDM of neutron and other particles have caused the fall of many theories to explain CP violation [24].

In 1987, when I first met Norman Ramsey (Figure 1), I asked him if he thought that a neutron EDM would be found. He replied that since we know that CP is violated, it should exist "unless nature is particularly malevolent against Norman Ramsey". He continued to point out that it was less clear whether it would be found during his lifetime. Twenty years later, particle EDMs remain elusive, but other examples of CP violation have been observed, in the decay of neutral B mesons $[25,26]$.

\subsection{Electric Dipole Moments of Particles, Nuclei and Atoms}

An atomic EDM experimentally can arise in a number of different ways, both through possible $\mathrm{P}$ and $\mathrm{T}$ violating interactions and through EDMs of fundamental particles [27]. A limit for $\mathrm{P}$ and $\mathrm{T}$ violating interactions between electrons and nucleons can be established from experimental limits of atomic EDMs combined with theoretical calculations the ratio between the induced atomic EDM and the relevant interaction parameters [28]. 


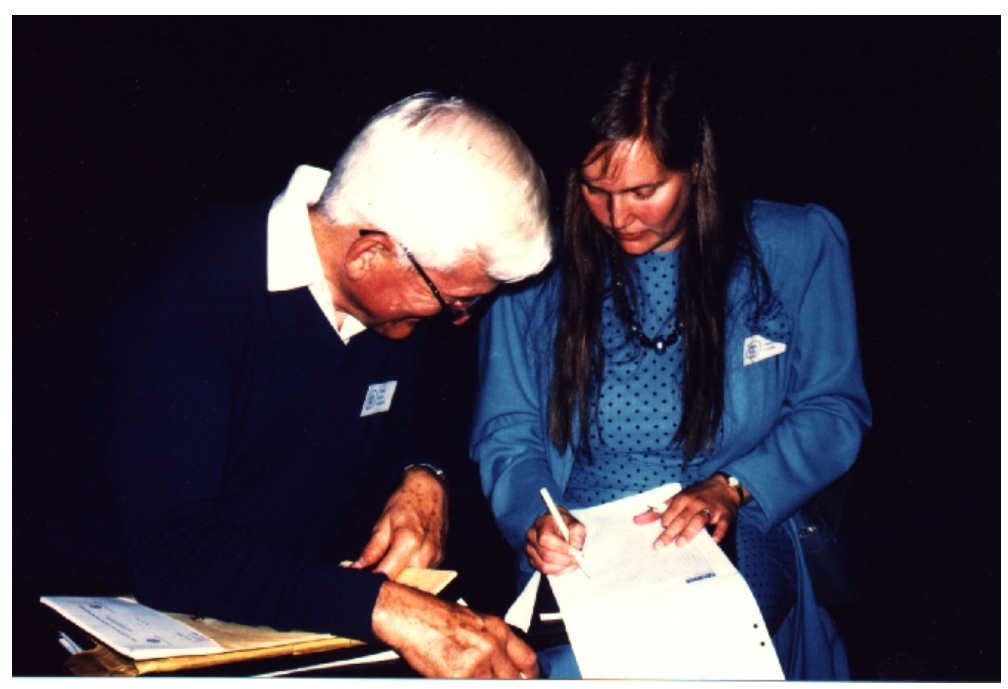

Fig. 1. Norman Ramsey together with the author discussing Tl isotope shifts. (Photo: Ingvar Lindgren)

In the limit of non-relativistic point particles, no atomic electric dipole moment can be induced by an EDM of a charged particle. However, through relativistic effects an electron EDM can induce an atomic EDM, as much as 100-1000 times larger in easily polarizable systems, such as Cs and $\mathrm{Tl}[29,30]$. The finite extension of the nucleus makes it possible, e.g. for a proton EDM, $d_{p}$, to cause an atomic EDM if the nuclear charge and dipole distributions differ. The observable effect depends on "Schiff moments", proportional to $\left(\left\langle r_{d}^{2}\right\rangle-\left\langle r_{c}^{2}\right\rangle\right)$ [31]. Schiff moments have to be obtained from nuclear theory and can also include additional contributions from $\mathrm{P}$ and $\mathrm{T}$ violating nucleon-nucleon interactions. In section 2.3, we show how observed hyperfine anomalies in $\mathrm{Tl}$ give a measure for the change in the difference between the nuclear charge and magnetic moment radii.

\subsubsection{Polar Molecules}

Experiments have also been performed for polar molecules, following the observation by Sandars that "a polar molecule can be almost completely polarized in attainable laboratory electric fields" making it possible to obtain large effective electric fields at the nucleus, which "will no longer average to zero, but will be parallel to the external field" [32]. Stringent experimental limits have been obtained for $\mathrm{TlF}[33,34,35,36,37]$, and $\mathrm{YbF}[38,39]$. Quantum chemists have risen to the challenge of applying relatively complete computational methods inlcuding a relativistic treatment also for such complex systems as TIF [40, 41], YbF [42] and PbO [43].

\section{Nuclear Effects in Atomic Spectra}

The nucleus affects atomic spectra in many ways. A well-known example is the hyperfine interaction between electrons and the nuclear magnetic moment, which results in a small splitting of spectral lines. The $21 \mathrm{~cm}$ hyperfine transition in the ground state of hydrogen provides the universal length scale for the plaque sent out with Pioneer. The hyperfine structure of the ground state of ${ }^{133} \mathrm{Cs}$ defines the second in the SI system of units. Accurate measurements of atomic hyperfine structure have been available since the 1950s, through the development of radio-frequency techniques [44].

Both the hyperfine interaction and the parity non-conserving interactions are sensitive to the electronic wave functions close to the nucleus, which depend on the nuclear charge distribution, which are, 
in general, relatively well known. However, the search for parity non-conserving effects raises a need for increased understanding of nuclear structure, including nuclear distributions other than those traditionally studied. The electroweak electron-nucleus interaction involves the neutron distribution, and the search for possible P and T violating effects in the nucleus involves so-called "Schiff moments". These distributions and moments must often be obtained from nuclear theory, and any additional experimental information may provide useful calibration for these calculations. One example is the hyperfine anomaly, resulting from isotopic differences in both charge and magnetization distribution, discussed more in Sections 2.1 and 2.3.

\subsection{Nuclear Charge Distributions}

The early theorists, without access to computers, had strong reasons to use analytical descriptions of charge distributions and potentials, that enabled series expansions of analytical solutions of the wavefunctions within and close to the nucleus. A common choice was the homogeneous charge distribution inside a radius $R=R_{0} A^{1 / 3}$, where $A$ is the mass number of nucleus. The most important parameter for many properties is the expectation value $\left\langle r^{2}\right\rangle$, which has the value $3 R^{2} / 5$ for a homogeneous nucleus. Already this simple distribution gives the correct analytical behaviour of the electronic wavefunctions at $r=0$ and provides useful expansions for a general understanding of the effects involved.

Experimental information about charge distributions are derived from many sources, including electron scattering. The experimental data indicate that $R_{0} \simeq 1.2 \mathrm{fm}$ gives a reasonable approximation for the homogeneous distribution. Clearly, the tail of a real nucleus is less sharp than indicated by the homogeneous distribution. The tail is often described in terms of a "skin thickness", $t$, defined as the distance in which the charge density falls from $90 \%$ of its central value to $10 \%$. Experiments indicate that $t$ is about $2.3 \mathrm{fm}$ for most nuclei.

The results from electron scattering experiments and are often expressed in terms of parameters for a Fermi-distribution [45], which gives a realistic description of the nuclear distribution [46, 47], and at the same time provides considerable flexibility in the analysis:

$\rho(r)=\frac{\rho_{0}}{1+\mathrm{e}^{(r-c) / a}}$

where, $c$ is the half-density radius and $a$ is related to the skin thickness as $t=(4 \ln 3) a$. For vanishing skin thickness, the Fermi form reduces to the homogeneous distribution with a radius $R=c$.

Another source of information are muonic X-ray energies. These probe somewhat different moments of the nuclear distribution, the so-called "Barrett moments", $\left\langle r^{k} e^{-\alpha r}\right\rangle$. Nevertheless, the results are quoted also in terms of $\left\langle r^{2}\right\rangle$ [48].

Optical isotope shifts provide an important source of complementary information, in particular for chains of radioactive isotopes. It is found that the changes in charge radius along an isotope chain are, in general, smaller than indicated by the textbook formula above. The isotope shifts also reveal an "odd-even staggering" of the $\left\langle r^{2}\right\rangle$ values, providing evidence of nuclear shell structure [49, 50]. Spectacular recent applications include the precision determination of the "deuteron structure radius" from the hydrogen-deuterium isotope shift of the 1s-2s two-photon resonance [51] and the study of Li isotope shifts [52], further discussed by Gordon Drake at this meeting.

\subsection{Nuclear Charge Distribution and Electronic Wavefunctions}

Outside the nucleus, the potential is identical to that from a point nucleus: $-Z e^{2} / 4 \pi \epsilon_{0} r$. Close to the origin, the potential is essentially constant. This determines the behaviour of the orbitals for very small $r$, which is obtained by a series expansion of the coupled radial equations from the Dirac 
equation:

$$
\begin{aligned}
-\hbar c\left[\frac{\mathrm{d}}{\mathrm{d} r}-\frac{\kappa}{r}\right] G(r) & =[\varepsilon-V(r)] F(r), \\
\hbar c\left[\frac{\mathrm{d}}{\mathrm{d} r}+\frac{\kappa}{r}\right] F(r) & =\left[\varepsilon+2 m_{\mathrm{e}} c^{2}-V(r)\right] G(r),
\end{aligned}
$$

where $\kappa=\mp(j+1 / 2)$ for $j=l \pm 1 / 2, \varepsilon$ is the binding energy and $F(r)$ and $G(r)$ are the radial parts of the "large" and "small" components, respectively, of a relativistic wavefunction. (The sign relation in Eq. (2) between $F$ and $G$ is valid for the "ls-convention" for the coupling of spin and orbital angular momentum.)

Inside an extended nucleus the leading term in the upper component is $r^{l}$. For $j=l+1 / 2$ orbitals, the lower component carries an extra factor $r$, whereas for the $j=l-1 / 2$ orbitals, the lower component has a small term $r^{l-1}$ which dominates for very small $r$.

We note that atomic orbitals with $j=1 / 2$ (i.e. $\mathrm{s}$ and $\mathrm{p}_{1 / 2}$ orbitals) have an appreciable probability within the nucleus, where their density is essentially constant. The effect of the nuclear distribution on atomic properties then becomes proportional to the expectation value, $\left\langle r^{2}\right\rangle=\int r^{2} \rho(\mathbf{r}) \mathrm{dV} / \int \rho(\mathbf{r}) \mathrm{dV}$, of the nuclear distribution.

The dependence on $\left\langle r^{2}\right\rangle$ is well-known from studies of optical isotope shifts, which are changes in transition frequency between different isotopes of an element. The "field" or "volume" isotope shift is the part due to the different charge distributions and is to a first approximation expressed as $\delta \nu=$ $F \delta\left\langle r^{2}\right\rangle$, where $F=(2 \pi / 3)\left(Z e^{2} / 4 \pi \epsilon_{0}\right) \Delta|\Psi(0)|^{2}$.

For heavy nuclei, also the next terms in the expansion of the wavefunctions become significant. The electron density then varies within the nucleus and higher moments, $\delta\left\langle r^{4}\right\rangle, \delta\left\langle r^{6}\right\rangle, \ldots$ of the nuclear distribution contribute. These can be accounted for through "Seltzer" correction factors, $\kappa$ [53], which have been tabulated [54]. The correction factor for $\mathrm{Bi}$, e.g., is about 0.92 . The correction factors are of course not needed if the distribution is used directly in the calculation.

The energy of a system is only weakly influenced by details in the nuclear charge distribution. Even for $Z=100$, using an extended nuclear charge gives only an $0.1 \%$ reduction of the binding energy. Differences between different distributions are less than a percent of the correction itself. However, for atomic properties sensitive to the wavefunction close to the nucleus, the effect is much larger. Using a point charge, with the resulting unphysical behaviour of the wavefunction at $r=0$, then leads to overestimates by several percent for high $Z$.

An important observation for the study of nuclear properties is that the orbital behaviour within the nucleus depends on the angular momentum, but is essentially independent of the binding energy. For very heavy nuclei, the binding energies are a larger fraction of the nuclear potential energy, giving a small $n$-dependence in the orbital behaviour close to the nucleus, as demonstrated e.g. by Shabaev [55] for hyperfine structure corrections for different values of $Z$.

For a many-electron system, all electrons are affected, but mainly through their interaction with the modified $j=1 / 2$ orbitals. It is then the sensitivity of the interaction of the $j=1 / 2$ orbitals that needs to be studied in detail.

The effect of the nuclear distribution can be parameterized in terms of the moment $\left\langle r^{2}\right\rangle,\left\langle r^{4}\right\rangle$ and $\left\langle r^{6}\right\rangle$ of the nuclear charge. To facilitate comparisons between different calculations, we therefore suggest that whatever nuclear distribution is used, these moments should be given (or sufficient information provided to make their extraction easy). 


\subsection{Hyperfine Anomalies and the Importance of Charge and Magnetization Distributions}

The magnetic hyperfine structure (hfs) arises from a coupling between the magnetic moments of the nucleus and the electron, given by

$h_{\mathrm{p}}^{\mathrm{hfs}}=\hat{\mathbf{r}} \cdot \frac{\mu \times \alpha}{r^{2}}$

for a point magnetic dipole.

The magnetic hyperfine interaction (Eq. 3) leads to an energy splitting which is often expressed as $A \mathbf{I} \cdot \mathbf{J}$, where $\mathbf{I}$ and $\mathbf{J}$, are the angular momenta of the nucleus and the electron, respectively. The hfs was an early source of information about nuclear spin and magnetic moments. In general, magnetic moments are known better through other sources, such as NMR. Hyperfine structure can thus be used in order to test atomic wavefunctions [56].

The ratio between the magnetic moment and the " $A$-factor" is, however, found to vary slightly between various isotopes of an element. This difference is expressed in terms of an"hyperfine anomaly":

${ }^{1} \Delta^{2} \equiv \frac{A_{1} g_{2}}{A_{2} g_{1}}-1$

Part of the hyperfine anomaly arises from differences in the charge distribution (the so-called BreitRosenthal effect $[57,58,59])$. Another contribution to the hyperfine anomaly, the "Bohr-Weisskopf effect" [60] arises from the distribution of nuclear magnetic moment, which leads to a modification of the hyperfine interaction (3) within the nucleus. In this way, the hyperfine anomaly can give information about differences in the magnetic moment distribution.

\subsection{Isotope Shifts}

The change, $\delta\left\langle r_{c}^{2}\right\rangle^{A A^{\prime}}$, in charge radius between two isotopes contributes to the differences in transition energies between the isotopes $A$ and $A^{\prime}$. The transition energies are also sensitive to the mass differences between different isotopes, due to the kinetic energy of the nucleus. The resulting isotope shifts can be written in the form [61]

$\delta \nu^{A A^{\prime}}=\left(K^{\mathrm{NMS}}+K^{\mathrm{SMS}}\right)\left(\frac{1}{M_{A}}-\frac{1}{M_{A}^{\prime}}\right)+F \kappa \delta\left\langle r^{2}\right\rangle^{A A^{\prime}}$

To provide a scale relating the observed isotope shifts to the desired $\delta\left\langle r_{c}^{2}\right\rangle^{A A^{\prime}}$ values, it is necessary to calculate or estimate the specific mass shift constant, $K^{\mathrm{SMS}}$, as well as the factor $F \kappa$ for the field shift, discussed in Sec. 3.2. From a computational point of view, it is often convenient to deal directly with shift of individual levels [62], which can be defined as the shift of the binding energy. Using many-body perturbation theory, starting with a closed-shell core as a reference, this quantity is obtained directly [63], without the need to "determine the weight of a captain by weighing the ship before and after he enters the ship".

For heavy systems the mass shift is very small. In the case of the $7 \mathrm{~s}-7 \mathrm{p}_{3 / 2}$ transition in Fr, $K^{\mathrm{NMS}}=m_{\mathrm{e}} \nu=228.99 \mathrm{GHz}$ u, the normal mass shift is of the order half a percent of the total isotope shift (about $5 \mathrm{MHz}$ for $\Delta A=1$ ). The specific mass shift arises from a correlation of the electronic momenta through the motion of the nucleus. A Dirac-Fock evaluation including the expectation value of $\mathbf{p}_{1} \cdot \mathbf{p}_{2}$ between the valence electron and the core, but also the effect of the perturbation of the core orbitals, gives a ratio SMS/NMS $\approx-2.4$ for the $7 s-7 p_{3 / 2}$ transition. This result will be modified by correlation effects, expected to reduce the SMS, as indeed found in the recent calculations by Dzuba et al [64], who obtain SMS/NMS $\approx-1.4$. For a relativistic system, the expressions for the mass shift should be modified, but this has so far been neglected in view of the small size of the effect. 


\subsection{The Field Isotope Shift}

The "field" or "volume" isotope shift arises due to the different nuclear potentials resulting from the different charge distributions. The field shift is sensitive to the change in electron density at the nucleus between the two states involved in the transition. A first approximation of the field shift constant is $F=(2 \pi / 3)\left(Z e^{2} / 4 \pi \varepsilon_{0}\right) \Delta|\Psi(0)|^{2}$. For a heavy nucleus, the electron density varies within the nucleus, and also higher moments, $\delta\left\langle r_{c}^{4}\right\rangle, \delta\left\langle r_{c}^{6}\right\rangle, \ldots$ contribute. The "Seltzer" correction factor [53], $\kappa$, in Eq. 4 has a value of about 0.93 for Fr [54]. The approach used in our calculations is to replace the contact interaction by an alternative operator that includes the radial dependence of the correction [65].

In the absence of theoretical values, the field shift constant for an s electron is often estimated from the s-electron hyperfine structure. Using the relations between the non-relativistic operators gives

$F_{n s}=-D Z A_{n s} / g_{I}$

where

$D=\left(1 \mathrm{fm} / \mathrm{a}_{0}\right)^{2} /\left[\alpha^{2}\left(m_{e} / 2 M_{p}\right)\left(\mu_{e} / \mu_{B}\right)\right]$,

which has the numerical value $6.16381 \times 10^{-3}$ if the A factor is given in $\mathrm{MHz}, g_{I}$ in nuclear magnetons and $\mathrm{F}$ in $\mathrm{MHz} / \mathrm{fm}^{2}$. The factor $\left(\mu_{e} / \mu_{B}\right)$ accounts for the anomalous magnetic moment of the electron.

Corrections to the ratio can arise from several sources. Most important is the modification due to the significantly larger relativistic correction factor for the field shift. For a many-electron system, the different angular structures of the operators lead to differences in the corrections from the manyelectron interactions, as analysed, e.g. in connection with calculations for K [66] and Fr [67].

\section{Atomic Theory}

\subsection{Hyperfine Structure in Alkali-Like Systems}

Hyperfine structures of alkali-like systems are an old application of Many-Body-Perturbation Theory (MBPT) with many accurate experimental results available for comparison [44], giving an indication of the accuracy to be expected.

The starting point for our calculations is usually a Dirac-Fock description of the closed-shell core. The Dirac-Fock potential from the core orbitals is then used to generate the valence orbital, as well as excited orbitals. Evaluating the effect of the hyperfine interaction on the valence orbital, itself, often gives a qualitatively correct description. A Dirac-Fock valence orbital, e.g., gives about $60-70 \%$ of the ground state hyperfine structure in the alkali atoms. For the iso-electronic singly ionized alkaline earth elements, the agreement is, of course, better, about $70-80 \%$ for the ground state. The situation is, however, quite different for cases where the hyperfine structure is inverted or strongly perturbed.

A first correction is often obtained by noting that the additional perturbation affects each electron, leading to a modification of the the potential felt by the other electrons. Keeping terms to lowest order in the external perturbation in the construction of the Dirac-Fock potential leads to a set of coupled equations which can be solved by iteration $[68,69,70]$ in procedures closely related to the "randomphase approximation" (RPA). For the ground states in the alkalis, these RPA-type calculations [69, 71] reproduce about $75-85 \%$ of the experimental values, improved to about $90 \%$ for the alkaline earth ions. In some cases, like the $4 \mathrm{~d}_{5 / 2}$ states in $\mathrm{Rb}[72,73]$ and $\mathrm{Sr}^{+}$, the RPA terms lead to an inversion of the hyperfine structure. In these cases, correlation effects are clearly crucial, and have been dealt with to higher orders.

Methods to treat single-particle effects to all orders, were applied to the study of parity nonconserving effect from early days $[74,75]$, whereas a relativistic treatment of correlation took longer to develop. 


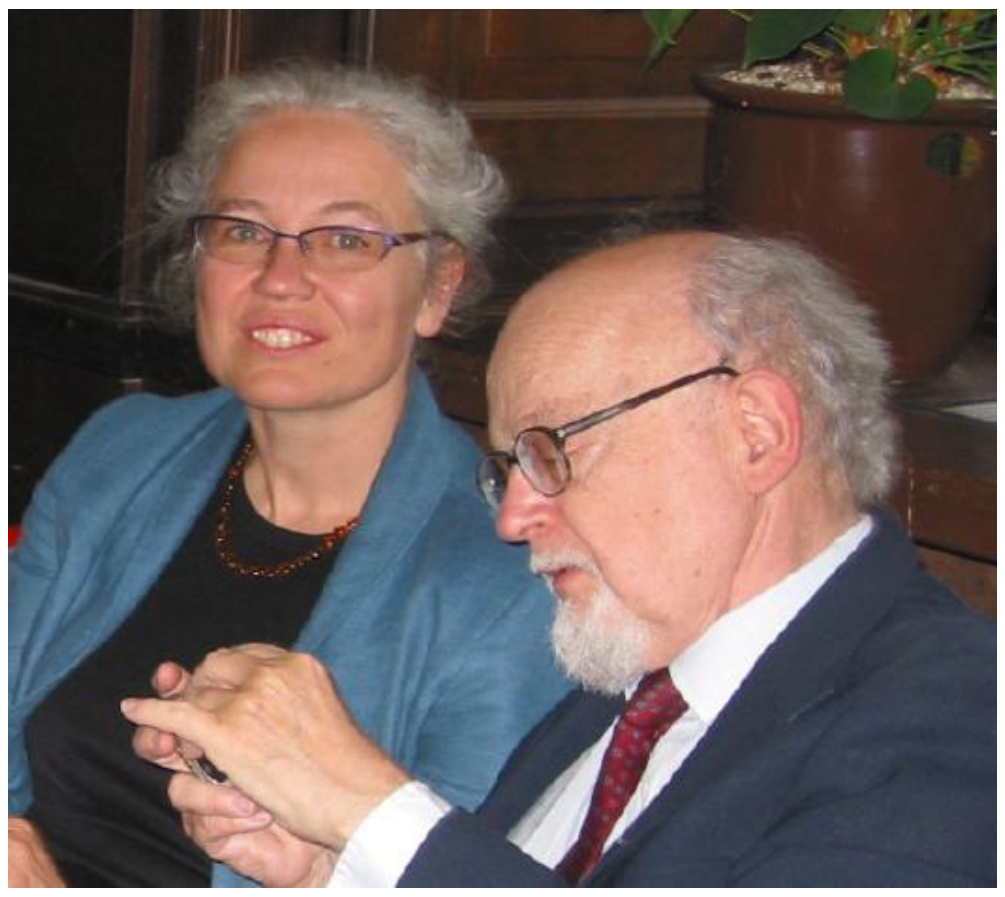

Fig. 2. Joe Sucher together with Eva Lindroth, June 2006)

\subsection{Atomic Theory Twenty Years Ago}

At the time of the first EBIT spectrum, whose twenty-year anniversary this conference celebrates, the ability to perform accurate calculation of atomic pair correlation was emerging. During the years to come, challenges both on the formal, numerical and computational level have been met, enabling theorists to deal with many different properties for heavy atoms, producing accurate numbers, valuable both in their own right and as tests to demonstrate the expected accuracy of calulcations where experimental data are not available for comparison.

Various types of non-relativistic methods had been developed to deal with correlation in different systems, including variational methods, such as CI (configuration interaction) and MCHF (MultiConfigurational Hartree-Fock) [76] and perturbational methods, such as MBPT (many-body perturbation theory) and Coupled-Cluster (CC) methods [77]. In general, variational methods were employed mostly for overall spectra and oscillator strengths, whereas perturbation approaches were often applied to properties resulting from interactions close to the nucleus, such as hyperfine structure and isotope shifts, which do not necessarily give large effects on the total energy.

Twenty years ago, many physicists working on atomic calculations met at a symposium in Trieste. Helium-like systems were a natural first step to inclusion of correlation effects for a many-electron system. For these systems, the very accurate theoretical results by Drake [78] provide a benchmark.

During the Trieste meeting a few groups presented results of their efforts to treat relativistic pair correlation, both on a formal and computational level. Although Dirac-Fock (DF) and Multiconfiguration Dirac-Fock (MDCF) [79, 80] in general gave good results for many properties, doubts had been cast on the fundament for these calculations [81]. The Dirac equation, used to obtain a relativistic wavefunction for the electron, has solutions both for positive and negative energies, where the negative energy solutions are seen as describing positrons. Mathematically, a complete set of solutions to the 
Dirac equation includes both types. For a two-electron system, there is an infinite number of combinations of one positive and one negative energy electron for any given sum of one-electron energies. If perturbation theory is applied without caution, the denominator will be zero for an infinite number of combinations, resulting in a "continuum dissolution". In a variational theory, the presence of these states may lead to a "variational collapse". In practice, the problem may be of limited importance, where boundary conditions restrict allowed solutions.

Sucher (Figure 2) advocates the application of projection operators [81]. However, even then, caution is required. The distinction between positive and negative energy one-electron functions depends on the potential used in the equation to define the orbital basis. It is a matter of preference if a perturbation is seen as modifying the basis set or as leading to admixtures of virtual electron-positron states [82, 83]. Hardekopf and Sucher [84] found that a projection of the nuclear potential onto positive free-electron states caused a lowering of the $1 \mathrm{~s}$ electron ground state of hydrogen-like ions. This illustrates that the projection onto free-electron positive energy states in fact led to inclusion of negative energy states of the hydrogen-like system [85].

Implementation of projection operators directly in the equations [86] leads to additional complications. Most calculations use instead an explicit summation over orbitals obtained with boundary conditions allowing only electronic states $[87,88,89]$. As in the case of non-relativistic calculations, the summation over angular momenta must be truncated. However, the relativistic corrections exhibit a slower angular convergence, $l^{-2}$, compared to $l^{-4}$ for the dominating non-relativistic term. For increased precision, the angular extrapolation may be performed separately for the relativistic correction [87]. The slow convergence is caused by the cusp for $r_{1}=r_{2}$, made even sharper by the application of projection operators, whether explicit or not.

Recent developments have aimed for a merging between many-body perturbation theory and QED $[90,91]$.

With the ability to treat pair correlation in a relativistic framework, the difficulties in the generalization to many-electron systems are very similar to the non-relativistic case, although the computational demands grow larger due to the need to treat two components for every orbital, and two j-values for every angular momentum (except s). A few examples of calculations for many-electron systems are presented in the next section.

\section{Numerical Results}

In this section, a few examples of numerical results are presented as illustrations. The first example is a calculation of the hyperfine structure of the $4^{2} D$ state of rubidium-like strontium. This state is of interest as a possible clock transition, and the calculations were performed to assist the search for the transition. The second example is a comparison of many-body effects on hyperfine structure and isotope shifts of the ground state of Fr. The numerical results enable more accurate extractions of isotope differences in nuclear charge radii. Finally, experimental and theoretical results for hyperfine anomalies in neutral $\mathrm{Tl}$ are combined with results for hydrogenlike $\mathrm{Tl}$, and gives a comparison between changes in nuclear magnetization and radii.

\subsection{The Hyperfine Splitting of the $4^{2} D$ state of $\mathbf{S r}^{+}$}

Single, trapped ions have come to play an important role as possible frequency standards, with several different candidate ions. One such ion is $\mathrm{Sr}^{+}$, which is studied by the NPL [92, 93]. A possible improvement might involve the odd isotope ${ }^{87} \mathrm{Sr}$, which has the nuclear spin $9 / 2$. The feasibility depends on the size of the hyperfine structure of the $4 \mathrm{~d}_{5 / 2}$ state, and a coupled-cluster calculation was performed to provide guidance in the search.

For the hyperfine structure of this state, several large contributions cancel partially and correlation effects were found to be extremely important - a few times larger than the final result [94]. Similar nonrelativistic calculations were performed for $\mathrm{Rb}$ [95]. 
The sensitivity to perturbations of the higher angular momentum states, in general, is related to the centrifugal term (given by $\hbar^{2} l(l+1) / 2 m r^{2}$ in the non-relativistic formalism). Griffin et al [96] have studied effective quantum numbers, $n^{*}$, for $d$ and $f$ electrons as a function of atomic number. They found $n^{*}$ to change smoothly with increasing atomic number, until it drops abruptly going from the inert gas to the alkali atom, and then even further reaching the alkaline earth. This "orbital collapse" results when the nuclear charge is sufficiently strong to pull part of the orbital inside the centrifugal barrier [97, 96]. The "effective potential" for the 4d electron in $\mathrm{Rb}^{\circ} \mathrm{Sr}^{+}$is the result of a subtle balance between the nuclear attraction, the repulsion from the core electrons and the centrifugal term. Corrections due to correlation effects can then have large effects, and often leads to a significant contraction of the valence orbital [72]. For the $4 \mathrm{~d}$ states in $\mathrm{Rb}$, this "Brueckner orbital" (BO) modification doubled the $\left\langle r^{-3}\right\rangle$ value for the valence electron, whereas the increase for the $4 \mathrm{~d}$ states in $\mathrm{Sr}$ is only about 25\%. Following the calculation of the hyperfine splitting in the $4 d_{5 / 2}$ state of $\mathrm{Sr}^{+}$, it has now been found and measured $[98,99]$ - within the theoretical uncertainty. The accurate theoretical results can also be used to extract nuclear quadrupole moments. A number of additional calculations have been performed more recently [100,101, 102].

\subsection{Many-Body Effects and Nuclear Charge Distributions in Francium}

Isotope shifts have been studied for long chains of isotopes, making possible systematic studies of isotopic differences in nuclear charge distributions. Early analyses of the experimental results were based on Hartree-Fock calculations by Bauche $[103,104]$. Many-body perturbation theory was first applied to the study of isotope shift of $\mathrm{Li}, \mathrm{K}$ [63], $\mathrm{Ne}$ [105], $\mathrm{Na}$ [65], $\mathrm{He}[106]$ and $\mathrm{Ca}[107,108]$.

After the development of relativistic methods, also heavier atoms could be studied, such as $\mathrm{Yb}$ [109], $\mathrm{Tl}$ [110], and Fr [111]. More recently Dzuba et al [64] performed an analysis of the isotope shifts for alkali-like systems.

We describe here briefly the results for the $7 \mathrm{~s}$ and $7 \mathrm{p}$ states in $\mathrm{Fr}$, as an illustration of similarities and differences between the higher-order corrections for hyperfine structure and isotope shifts. Francium has been suggested as a candidate system to study isotope-dependent parity non-conservation [112] Isotope shifts in the range ${ }^{207} \mathrm{Fr}$ to ${ }^{228} \mathrm{Fr}$ were measured in the mid-eighties at ISOLDE $[113,114]$ and recent accurate hyperfine anomaly measurements may give a handle on neutron distributions [112].

The relativistic coupled cluster calculations, $[111,115]$ included pair excitations from core orbitals down to $5 \mathrm{~s}$ and excitations up to $l=5$. Using these wavefunctions to evaluate properties gives several corrections to the Dirac-Fock single-particle matrix element. Some of the many-body corrections differ between various operators, due to their different angular structure $[66,111]$.

The ratio between the field shift constant and the hyperfine structure of an s state can be changed if corrections to the wavefunctions affect the two properties differently. The DF ratio remains unchanged when Brueckner type corrections are added, since these are in effect a modification of the orbital. For both properties the Brueckner orbital corrections bring an increase of $42 \%$ for the $7 \mathrm{~s}$ state in Fr.. However, the screening effects ("RPA" type corrections) differ: The hyperfine structure is sensitive only to the spin density at the nucleus, whereas the field shift is represented by a scalar operator and is sensitive to the total electron density. An all-order treatment of the screening was found to add $9 \%$ and 3\%, respectively to the Brueckner orbital value for the hyperfine structure and field shift constants of the $7 \mathrm{~s}$ state. The different angular structure of the operators also modifies the detailed correlation effects, although, in general, these are often smaller than the BO corrections, in the case of Fr bringing a reduction of about $3 \%$ for both operators.

The imperfect analogy between the FS and hfs for s-states illustrates that different operators, with different angular structures, probe different aspects of the interaction between valence and core orbitals. This is, of course, true also of nuclear densities, and it can thus not be taken for granted that the nuclear charge distribution gives an adequate description for other properties.

The calculated hyperfine structure, $\mathrm{A}(7 \mathrm{~s}) / \mathrm{g}_{I}=10002 \mathrm{MHz} / \mu_{N}$, giving $A\left({ }^{211} \mathrm{Fr}\right)=8882 \mathrm{MHz}$, 
slightly larger than the experimental value $8713.9(8) \mathrm{MHz}$, although still within the error bars arising from the uncertainty in the nuclear magnetic moment, $\mu\left({ }^{211} \mathrm{Fr}\right)=4.00(9) \mu_{N}$. From the comparison of the $A$-factors, we expect that the final field shift constant, $F\left(7 s-7 p_{3 / 2}\right)=-21.0 \mathrm{GHz} / \mathrm{fm}^{2}$ (including the correction $\kappa$ to allow for higher moments), should have an uncertainty of about $2 \%$, which dominates the uncertainty of the changes, $\delta\left\langle r^{2}\right\rangle$, in charge radii obtained from optical isotope shifts $[113,114]$.

More recently Dzuba et al [64] performed an analysis of the IS for alkali-like systems, including Fr. They included a partial summation of three-particle correlation effects, and obtained a field shift constant of $F\left(7 s-7 p_{3 / 2}\right)=-21.8 \mathrm{GHz} / \mathrm{fm}^{2}$, with an estimated uncertainty of $1 \%$.

These calculated field shift constants lead to revised values, of $\delta\left\langle r^{2}\right\rangle$ in Fr, slightly larger than earlier tabulations. The results illustrate how atomic calculations in combination with accurate experiments can provide information about other properties, including nuclear structure. In the study of highly charged hydrogen-like systems, more detailed knowledge of the nuclear charge distribution makes possible more stringent comparisons between theory and experiment, for energies and hyperfine structure, discussed in the next section.

\subsection{Hyperfine Anomalies and the Bohr Weisskopf Effect, for ${ }^{203} \mathbf{T l}$ and ${ }^{205} \mathbf{T l}$}

The study of highly ionized systems shifts the focus to QED effects. For hydrogen-like systems, no atomic correlation is present. However, in order to provide an accurate comparison with calculated radiative corrections, it is important that the influence of nuclear structure is well understood.

Measurements of hfs for highly-charged hydrogen-like systems have been reported for several elements: ${ }^{165} \mathrm{Ho}^{66+}$ [116], ${ }^{185} \mathrm{Re}^{74+}$ [117], ${ }^{187} \mathrm{Re}^{74+}$ [117], ${ }^{207} \mathrm{~Pb}^{81+}$ [118], ${ }^{209} \mathrm{Bi}^{82+}$ [119] and ${ }^{209} \mathrm{Bi}^{80+}$ [120]. The results, in particular for ${ }^{165} \mathrm{Ho}^{66+}$ and ${ }^{185,187} \mathrm{Re}^{74+}$, were found to differ considerably from predictions based on a single-nucleon description for the nuclear magnetization [55, $121,122]$. For the case of Tl, which is closer to a double-magic nucleus, better agreement should be expected.

However, without resorting to nuclear theory, the isotopic difference in hfs for the hydrogen-like systems can be predicted by using hyperfine anomalies for neutral $\mathrm{Tl}$ [110, 122], which were measured in the early days of radiofrequency spectroscopy. For the $6 \mathrm{p}_{1 / 2}$ ground state of neutral Tl, the anomaly is ${ }^{203} \Delta^{205}=-1.036(3) \times 10^{-4}$ [123]. Many-body effects were found to account for about $9 \%$ of the total $6 \mathrm{p}_{1 / 2}$ anomaly in neutral $\mathrm{Tl}$.

To estimate the size of the $1 \mathrm{~s}$ anomaly for the hydrogen-like system, we recall that a one-electron anomaly depends on the angular momentum, but is essentially $n$-independent. The ratio between the Bohr-Weisskopf effect for $\mathrm{s}_{1 / 2}$ and $\mathrm{p}_{1 / 2}$ orbitals is about 3.3. The total anomaly is, however, also affected by the nuclear charge distribution, which affects the orbitals close to the nucleus. The ratio between this "Breit-Rosenthal(-Crawford-Schawlow)" [57, 58, 59]) effect for $\mathrm{s}_{1 / 2}$ and $\mathrm{p}_{1 / 2}$ orbitals is slightly larger, 3.7. The ratio between the anomalies for $s_{1 / 2}$ and $\mathrm{p}_{1 / 2}$ thus depends on the relative importance of changes in the nuclear charge and magnetization distributions.

To find the importance of changes in the charge distributions, calculations were performed for orbitals obtained with several different choices of the parameters, $c$ and $a$, in the Fermi distribution for the charge distribution(1). The orbitals obtained for different distributions were then used to evaluate the hyperfine interacation. The "Breit-Rosenthal" effect can be expressed in terms of a correction factor $\left(1-\varepsilon_{\mathrm{BR}}\right)$, which can be parametrized in terms of changes in the charge distribution, using $\varepsilon_{\mathrm{BR}}=$ $-x_{r} \delta\left\langle r_{\mathrm{c}}^{2}\right\rangle$ (neglecting any changes in the skin thickness). For the interaction with an extended magnetic dipole moment, which gives less weight than a point dipole to the electron orbital for very small radii, we obtained the value $x_{r}=-0.78 \times 10^{-3} \mathrm{fm}^{-2}$ for the $1 \mathrm{~s}$ ground state of hydrogen-like $\mathrm{Tl}$ [4].

Muonic isotope shifts measurements [48] give $\delta\left\langle r_{\mathrm{c}}^{2}\right\rangle$ by assuming Fermi distributions with the same skin thickness, $a$. The result for the difference between ${ }^{205} \mathrm{Tl}$ and ${ }^{205} \mathrm{Tl}$ is $\delta\left\langle r_{\mathrm{c}}^{2}\right\rangle=0.115(3) \mathrm{fm}^{2}$, giving ${ }^{203} \Delta_{\mathrm{c}}^{205}=-0.897(23) \times 10^{-4}$ for $1 \mathrm{~s}$ in H-like Tl and ${ }^{203} \Delta_{\mathrm{c}}^{205}=-0.245(6) \times 10^{-4}$ for the single 
particle effect of $6 \mathrm{p}_{1 / 2}$ in neutral Tl. The rest of the anomaly is then ascribed to differences in nuclear magnetization. The theoretical estimates for the ratio between the effects for the $6 \mathrm{p}_{1 / 2}$ anomaly in neutral $\mathrm{Tl}$ and $1 \mathrm{~s}$ anomalies for hydrogen-like $\mathrm{Tl}$ can then be used to estimate the total anomaly in hydrogenlike $\mathrm{Tl}$.

Following these considerations, the data from the $6 \mathrm{p}_{1 / 2}$ ground state of neutral Tl yield an estimate of $-3.21(5) \times 10^{-4}$ for the anomaly of the 1s ground state of hydrogen-like $\mathrm{Tl}$, corresponding to an energy difference of $30.71 \pm 0.16 \mathrm{meV}$ or a wavelength difference of $36.17 \pm 0.19 \AA$, in excellent agreement with the experimental value [4]. The estimated difference in magnetization radius becomes $\delta\left\langle r_{\mathrm{m}}^{2}\right\rangle=0.38(1) \mathrm{fm}^{2}$, considerably larger than the change in charge radius.

\section{Conclusion}

The search for atomic parity non-conservation stimulated the development of atomic calculations. Fundamental problems were encountered during the development. The methods to treat pair correlation in a relativistic framework have come a long way since the early efforts in mid-eighties. These developments enable theorists to make useful predictions, and to interpret experimental results from atomic spectroscopy in terms of nuclear properties or interaction parameters. However, the extraction of parameters is sometimes limited by an incomplete understanding of the nuclear physics involved. Today many of the results obtained from a combination of atomic theory and experiment direct the challenge to nuclear theory. The study of atomic physics on the borderline to nuclear and particle physics brings together physicists with very different backgrounds, and is characterized by many-body interactions, both on the particle and personal level.

\section{Acknowledgements}

During recent years, my research focus has shifted from studies of atoms to individuals, who learn or discover physics in different contexts, including amusement parks. I very much appreciated the invitation to be part of the EBIT celebration and to share the excitement, and to have a chance again to experience the joy of being part of a generous and creative physics research community. My work has been supported over the years by the Swedish Natural Science Research Council (NFR), in recent years replaced by The Swedish Research Council (VR), who provided travel support for the conference.

\section{References}

1. T. D. Lee and C. N. Yang, Phys. Rev. 104, 254 (1956).

2. C. S. Wu, E. Ambler, R. W. Hayward, D. D. Hoppes, and R. P. Hudson, Phys. Rev. 105, 1413 (1957).

3. N. F. Ramsey and E. M. Purcell, Phys. Rev. 78, 807 (1950).

4. P. Beiersdorfer et al., Phys. Rev. A 64, 032506 (2001).

5. M. Gardner, The New Ambidextrous Universe : Symmetry and Asymmetry, from Mirror Reflections to Superstrings, W H Freeman \& Co, 1991.

6. M. A. Bouchiat and C. Bouchiat, Phys. Lett. 48B, 111 (1974).

7. S. Bennet and C. E. Wieman, Phys. Rev. Lett. 82, 2484 (1999).

8. C. S. Wood et al., Science 275, 1759 (1997).

9. S. A. Blundell, W. R. Johnson, and J. Sapirstein, Phys. Rev. A 43, 3407 (1991).

10. S. A. Blundell, A. C. Hartley, Z. W. Liu, A.-M. Mårtensson-Pendrill, and J. Sapirstein, Theoretica Chimica Acta 80, 257 (1991).

11. A. Derevianko, Phys. Rev. Lett. 85, 1618 (2000).

12. V. A. Dzuba, V. V. Flambaum, and M. S. Safronova, Physical Review A (Atomic, Molecular, and Optical Physics) 73, 022112 (2006).

13. V. M. Shabaev, K. Pachucki, I. I. Tupitsyn, and V. A. Yerokhin, Physical Review Letters 94, 213002 (2005).

14. V. A. Dzuba, V. V. Flambaum, and I. B. Khriplovich, Z. Phys. D 1, 243 (1986). 
15. E. N. Fortson, Y. Pang, and L. Wilets, Phys. Rev. Lett. 65, 2857 (1990).

16. S. J. Pollock, E. N. Fortson, and L. Wilets, Phys. Rev. C 46, 2587 (1992).

17. J. James and P. G. H. Sandars, J. Phys. B 32, 3295 (1999).

18. Y. B. Zel'dovich, Sov. Phys. JETP 6, 1184 (1958).

19. I. B. K. V. V. Flambaum and O. P. Sushkov, Physics Letters B, 146, 367 (1984).

20. S. C. Bennett and C. E. Wieman, Phys. Rev. Lett. 82, 2484 (1999).

21. E. Gomez, S. Aubin, G. D. Sprouse, L. A. Orozco, and D. P. DeMille, Physical Review A (Atomic, Molecular, and Optical Physics) 75, 033418 (2007).

22. L. L, Nuclear Physics 3, 127 (1957).

23. J. H. Christenson, J. W. Cronin, V. L. Fitch, and R. Turlay, Phys. Rev. Lett. 13, 138 (1964).

24. N. F. Ramsey, Reports on Progress in Physics 45, 95 (1982).

25. B. Aubert et al., Phys. Rev. Lett. 87, 091801 (2001).

26. K. Abe et al., Phys. Rev. Lett. 87, 091802 (2001).

27. O. Sushkov, V. V. Flambaum, and I. B. Khriplovich, Sov. Phys. JETP 60, 873 (1984).

28. A.-M. Mårtensson-Pendrill, Phys. Rev. Lett. 54, 1153 (1985).

29. P. G. H. Sandars, Physics Letters 14, 194 (1965).

30. P. G. H. Sandars, Physics Letters 22, 290 (1966).

31. L. I. Schiff, Phys. Rev. 132, 2194 (1963).

32. P. G. H. Sandars, Physical Review Letters 19, 1396 (1967).

33. E. A. Hinds and P. G. H. Sandars, Phys. Rev. A 21, 480 (1980).

34. D. A. Wilkening, N. F. Ramsey, and D. J. Larson, Phys. Rev. A 29, 425 (1984).

35. D. Schropp, D. Cho, T. Vold, and E. A. Hinds, Phys. Rev. Lett. 59, 991 (1987).

36. D. Cho, K. Sangster, and E. A. Hinds, Phys. Rev. Lett. 63, 2559 (1989).

37. D. Cho, K. Sangster, and E. A. Hinds, Phys. Rev. A 44, 2783 (1991).

38. B. E. Sauer, J. Wang, and E. A. Hinds, The Journal of Chemical Physics 105, 7412 (1996).

39. E. A. Hinds, Physica Scripta 1997, 34 (1997).

40. J. K. Laerdahl, T. Saue, K. Fægri, and H. M. Quiney, Phys. Rev. Lett. 79, 1642 (1997).

41. A. N. Petrov et al., Phys. Rev. Lett. 88, 073001 (2002).

42. H. M. Quiney, H. Skaane, and I. P. Grant, Journal of Physics B: Atomic, Molecular and Optical Physics 31, L85 (1998).

43. T. A. Isaev et al., Physical Review A (Atomic, Molecular, and Optical Physics) 69, 030501 (2004).

44. E. Arimondo, M. Inguscio, and P. Violino, Reviews of Modern Physics 49, 31 (1977).

45. H. de Vries, C. W. de Jager, and C. de Vries, At. Data Nucl. Data Tables 36, 495 (1987).

46. R. Hofstadter, H. R. Fechter, and J. A. McIntyre, Physical Review 92, 978 (1953).

47. B. Hahn, D. G. Ravenhall, and R. Hofstadter, Physical Review 101, 1131 (1956).

48. R. Engfer, H. Schneuwly, J. L. Vuilleumier, H. K. Walter, and A. Zehnder, Atomic Data and Nuclear Data Tables 14, 509 (1974).

49. P. Aufmuth, K. Heilig, and A. Steudel, Atomic Data and Nuclear Data Tables 37, 455 (1987).

50. E. W. Otten, Treatise on Heavy-Ion Science 8, 517 (1989).

51. A. Huber et al., Physical Review Letters 80, 468 (1998).

52. R. Sanchez et al., Physical Review Letters 96, 033002 (2006).

53. E. C. Seltzer, Phys. Rev. 188, 1916 (1969).

54. G. Torbohm, B. Fricke, and A. Rosén, Phys. Rev. A 31, 2038 (1985).

55. V. M. Shabaev, Journal of Physics B 27, 5825 (1994).

56. M. S. Safronova, W. R. Johnson, and A. Derevianko, Physical Review A 60, 4476 (1999).

57. J. E. Rosenthal and G. Breit, Physical Review 41, 459 (1932).

58. M. F. Crawford and A. L. Schawlow, Physical Review 76, 1310 (1949).

59. H. J. Rosenberg and H. H. Stroke, Physical Review A 5, 1992 (1972).

60. A. Bohr and V. F. Weisskopf, Physical Review 77, 94 (1950).

61. E. W. Otten, Treatise on Heavy-Ion Science 8, 517 (1989).

62. L. R. Pendrill and K. Niemax, J. Phys. B 13, L461 (1980).

63. A. M. Martensson and S. Salomonson, Journal of Physics B: Atomic and Molecular Physics 15, 2115 (1982).

64. V. A. Dzuba, W. R. Johnson, and M. S. Safronova, Physical Review A (Atomic, Molecular, and Optical 
Physics) 72, 022503 (2005).

65. E. Lindroth and A.-M. Mårtensson-Pendrill, Z. Physik. D 309, 277 (1982).

66. A.-M. Mårtensson-Pendrill, L. Pendrill, S. Salomonson, A. Ynnerman, and H. Warston, Journal of Physics B 23, 1749 (1990).

67. A.-M. Mårtensson-Pendrill, Molecular Physics 98, 1201 (2000).

68. S. Garpman, I. Lindgren, J. Lindgren, and J. Morrison, Z. Physik. A 276, 167 (1976).

69. J.-P. Desclaux, Int. J. Quant. Chem. 6, 25 (1972).

70. I. Lindgren and A.-M. Mårtensson, Phys. Rev. A 26, 3249 (1977).

71. J.-L. Heully and A.-M. Mårtensson-Pendrill, Physica Scripta 27, 291 (1983).

72. I. Lindgren, J. Lindgren, and A.-M. Mårtensson, Z. Physik A 279, 113 (1976).

73. I. Lindgren, J. Lindgren, and A.-M. Mårtensson, Phys. Rev. A 15, 2123 (1977).

74. P. G. H. Sandars, Journal of Physics B: Atomic and Molecular Physics 10, 2983 (1977).

75. A.-M. Mårtensson, E. M. Henley, and L. Wilets, Phys. Rev. A 24, 308 (1981).

76. C. F. Fischer, The Hartree-Fock Method for Atoms, John Wiley \& Sons, New York, 1977.

77. I. Lindgren, Physica Scripta 36, 591 (1987).

78. G. W. F. Drake, Nuclear Instruments and Methods in Physics Research Section B 31, 7 (1988).

79. J. P. Desclaux, Comput. Phys. Commun. 9, 31 (1975).

80. I. P. Grant, B. J. McKenzie, P. H. Norrington, D. F. Mayers, and N. C. Pyper, Comput. Phys. Commun. 21, 207 (1980).

81. J. Sucher, Phys. Rev. A 22, 348 (1980).

82. M. H. Mittleman, Phys. Rev. A 24, 1167 (1981).

83. J. Sapirstein, K. T. Cheng, and M. H. Chen, Phys. Rev. A 59, 259 (1999).

84. G. Hardekopf and J. Sucher, Phys. Rev. A 30, 703 (1984).

85. J.-L. Heully, I. Lindgren, E. Lindroth, and A.-M. Mårtensson-Pendrill, Physical Review A 33, 4426 (1986).

86. E. Lindroth, J. L. Heully, I. Lindgren, and A. M. Martensson-Pendrill, Journal of Physics B: Atomic and Molecular Physics 20, 1679 (1987).

87. S. Salomonson and P. Öster, Physical Review A 40, 5559 (1989).

88. H. M. Quiney, I. P. Grant, and S. Wilson, Journal of Physics B: Atomic, Molecular and Optical Physics 23, L271 (1990).

89. W. R. Johnson, S. A. Blundell, and J. Sapirstein, Phys. Rev. A 37, 307 (1988).

90. J. Sapirstein, Rev. Mod. Phys. 70, 55 (1998).

91. I. Lindgren, S. Salomonson, and D. Hedendahl, Physical Review A (Atomic, Molecular, and Optical Physics) 73, 062502 (2006).

92. M. G. Boshier, G. P. Barwood, G. Huang, and H. A. Klein, Appl. Phys. B 71, 51 (2000).

93. G. Barwood, K. Gao, P. Hill, G. H. G, and H. A. Klein, IEEE TRANSACTIONS ON INSTRUMENTATION AND MEASUREMENT 50, 543 (2001).

94. A.-M. Mårtensson-Pendrill, Journal of Physics B: Atomic, Molecular and Optical Physics 35, 917 (2002).

95. A.-M. Mårtensson, Atomic many-body calculations of pair correlation and of fine and hyperfine structure, Thesis, University of Göteborg, 1978.

96. D. C. Griffin, K. L. Andrew, and R. D. Cowan, Phys. Rev. 177, 62 (1969).

97. A. R. P. Rau and U. Fano, Phys. Rev. 167, 7 (1968).

98. G. P. Barwood, K. Gao, P. Gill, G. Huang, and H. A. Klein, Phys. Rev. A 67, 013402 (2003).

99. P. Gill et al., Measurement Science and Technology 14, 1174 (2003).

100. K. zhi Yu, L. jin Wu, B. cong Gou, and T. yun Shi, Physical Review A (Atomic, Molecular, and Optical Physics) 70, 012506 (2004).

101. W. M. Itano, Physical Review A (Atomic, Molecular, and Optical Physics) 73, 022510 (2006).

102. B. K. Sahoo et al., Physical Review A (Atomic, Molecular, and Optical Physics) 75, 042504 (2007).

103. J. Bauche, PhD thesis, Université de Paris, 1980, In French.

104. J. Bauche, J. Phys. (Paris) 35, 19 (1974), In French.

105. S. Hörbäck, A.-M.Mårtensson-Pendrill, S. Salomonson, and U. Österberg, Phys. Scr. 28, 469 (1983).

106. E. Lindroth and A.-M. Mårtensson-Pendrill, Z. Physik. D 316, 265 (1984).

107. E. Lindroth, A.-M. Mårtensson-Pendrill, and S. Salomonson, Phys. Rev. A 31, 58 (1985). 
108. A.-M. Mårtensson-Pendrill et al., Phys. Rev. A 45, 4675 (1992).

109. A.-M. Mårtensson-Pendrill, D. S. Gough, and P. Hannaford, Physical Review A 49, 3351 (1994).

110. A.-M. Mårtensson-Pendrill, Physical Review Letters 74, 2184 (1995).

111. A.-M. Mårtensson-Pendrill, Hyperfine Interactions 127, 41 (2000).

112. J. S. Grossman et al., Phys. Rev. Lett. 83, 935 (1999).

113. A. Coc et al., Phys. Lett. 163B, 66 (1985).

114. A. Coc et al., Nucl. Phys. A468, 1 (1987).

115. A.-M. Mårtensson-Pendrill, Mol. Phys. 98, 1201 (2000).

116. J. R. Crespo López-Urrutia, P. Beiersdorfer, D. W. Savin, and K. Widmann, Physical Review Letters 77, 826 (1996).

117. J. R. Crespo López-Urrutia et al., Physical Review A 57, 879 (1998).

118. P. Seelig et al., Physical Review Letters 81, 4824 (1998).

119. I. Klaft et al., Physical Review Letters 73, 2425 (1994).

120. P. Beiersdorfer, A. L. Osterheld, J. H. Scofield, J. R. C. López-Urrutia, and K. Widmann, Physical Review Letters 80, 3022 (1998).

121. V. M. Shabaev, M. Tomaselli, T. Kühl, A. N. Artemyev, and V. A. Yerokhin, Physical Review A 56, 252 (1997).

122. M. G. H. Gustavsson, C. Forssén, and A.-M. Mårtensson-Pendrill, Hyperfine Interactions 127, 347 (2000).

123. A. Lurio and A. G. Prodell, Physical Review 101, 79 (1956). 